\title{
Localization Operators and an Uncertainty Principle for the Discrete Short Time Fourier Transform
}

\author{
Carmen Fernández, Antonio Galbis, and Josep Martínez \\ Departamento de Análisis Matemático, Universidad de Valencia, Burjasot 46100, Valencia, Spain \\ Correspondence should be addressed to Carmen Fernández; fernand@uv.es
}

Received 9 December 2013; Accepted 2 January 2014; Published 13 February 2014

Academic Editor: Alfredo Peris

Copyright (C) 2014 Carmen Fernández et al. This is an open access article distributed under the Creative Commons Attribution License, which permits unrestricted use, distribution, and reproduction in any medium, provided the original work is properly cited.

Localization operators in the discrete setting are used to obtain information on a signal $f$ from the knowledge on the support of its short time Fourier transform. In particular, the extremal functions of the uncertainty principle for the discrete short time Fourier transform are characterized and their connection with functions that generate a time-frequency basis is studied.

\section{Introduction}

It is a well-known fact that a nontrivial function and its Fourier transform cannot be simultaneously well localized, and many variants of this vague statement are collected under the term uncertainty principle. We refer to [1] and the references therein for different versions. For functions defined on finite abelian groups, localization is generally expressed in terms of the cardinality of the support of the function. For instance, Donoho and Stark [2] proved the following: given a finite sequence $\left(x_{i}\right)_{i=1}^{d-1}$ with discrete Fourier transform $\left(\widehat{x}_{i}\right)_{i=1}^{d-1}$ let $N_{t}$ and $N_{w}$ be the cardinals of their supports, then

$$
N_{t} \cdot N_{w} \geq d
$$

Meshulam [3] obtained a generalization for nonabelian groups. Moreover, those $x$ for which one has the equality were characterized (see $[2,4,5])$. When $d$ is a prime number the result was improved by Tao [6], who showed that the sum of the number of nonzero entries in a finite sequence and the number of nonzero entries in its Fourier transform are strictly larger than $d$. An extension to abelian groups of finite order is given in [7].

Results of this type for joint time-frequency representations in the continuous case are obtained in [8-13] among others, and for functions defined on finite abelian groups in [14], where it is proved that the cardinality of the support of any short time Fourier transform of a nontrivial function defined on a finite abelian group is bounded below by the order of the group. Ghobber and Jaming obtained in [15] an uncertainty principle for the representation of a vector in two bases, which permitted in particular to get a quantitative version of the above-mentioned result by Krahmer et al. [14]. In Theorem 1 we present a different proof of the quantitative result in [15] improving the constant there obtained.

Our aim is to obtain information on a signal $f$ from the knowledge on the support of its short time Fourier transform. To this end we introduce the localization operators in the discrete setting, which provide a filtered version of the original signal $f$. The importance of localization operators in this context is due to the fact that, for a given subset $\Lambda$ of $\mathbb{Z}_{d} \times \mathbb{Z}_{d}, f=\left(f_{i}\right)_{i=0}^{d-1}$ is a fixed point of the localization operator $L_{\Lambda, g}$ with window $g=\left(g_{i}\right)_{i=0}^{d-1}$ whenever $V_{g} f$ is supported in $\Lambda$. In Theorem 3 we characterize those finite sequences $f$ and $g$ such that the cardinal of the support of $V_{g} f$ is minimum; that is, we characterize the extremal functions of the uncertainty principle for the discrete short time Fourier transform.

Additional relevant information about the functions can be derived from the support of the short time Fourier transform. For instance, it is easy to see that when $f$ and $g$ are periodic, the short time Fourier transform $V_{g} f$ is supported in a certain subgroup. We will see in Proposition 4 that a sort of converse also holds. Here again localization operators are 
used in the proof. In a recent paper, Gilbert and Rzeszotnik [16] calculated the norm of the Fourier transform from the $L^{p}$ space on a finite abelian group to the $L^{q}$ space on the dual group and they studied the points at which the norm is attained. In connection with this problem they consider the functions on the group such that the set of all their translations coincides (up to some constants) with the set of all their modulations and produce an orthonormal basis. In the case of cyclic groups, these functions are easily characterized (up to constants) by the support of $V_{f} f$ (Proposition 6). In particular, for groups of prime order these are essentially the extremal functions of the uncertainty principle for the discrete short time Fourier transform.

\section{The Results}

Let $\mathbb{Z}_{d}$ denote the cyclic group $\mathbb{Z}_{d}:=\mathbb{Z} / d \mathbb{Z}$ in which addition is performed modulo $d$. If $f: \mathbb{Z}_{d} \rightarrow \mathbb{C}$ is any complexvalued function on $\mathbb{Z}_{d}$, we define the Fourier transform $\widehat{f}$ : $\mathbb{Z}_{d} \rightarrow \mathbb{C}$ by the formula

$$
\widehat{f}(k)=\frac{1}{\sqrt{d}} \sum_{\ell=0}^{d-1} f(\ell) e^{-2 \pi i \ell k / d} .
$$

From now on, we identify $\mathbb{C}^{\mathbb{Z}_{d}}$ with $\mathbb{C}^{d}$ and we endow it with the Euclidean norm. The translation operator $T_{j}, j \in \mathbb{Z}_{d}$, is the unitary operator on $\mathbb{C}^{d}$ given by $\left(T_{j} f\right)(\ell):=f(\ell-j)$. Similarly, the modulation operator $M_{k}, k \in \mathbb{Z}_{d}$, is the unitary operator defined by

$$
\left(M_{k} f\right)(\ell):=e^{2 \pi i k \ell / d} f(\ell) .
$$

We have $\widehat{M_{k} f}=T_{k} \widehat{f}$. The short-time Fourier transform of $f \in \mathbb{C}^{d}$ with respect to the window $g \in \mathbb{C}^{d}$ is given by ([1719])

$$
\left(V_{g} f\right)(j, k)=\frac{1}{\sqrt{d}} \sum_{\ell=0}^{d-1} f(\ell) \overline{g(\ell-j)} e^{-2 \pi i \ell k / d}, \quad j, k \in \mathbb{Z}_{d} .
$$

That is,

$$
\left(V_{g} f\right)(j, k)=\left(\widehat{f \cdot \overline{T_{j} g}}\right)(k)=\frac{1}{\sqrt{d}}\left\langle f, M_{k} T_{j} g\right\rangle .
$$

It is well-known that

$$
\sum_{(j, k) \in \mathbb{Z}_{d}^{2}}\left|\left(V_{g} f\right)(j, k)\right|^{2}=\|f\|^{2}\|g\|^{2} .
$$

This identity means that $\left\{M_{k} T_{j} g:(j, k) \in \mathbb{Z}_{d}^{2}\right\}$ is a tight frame for $\mathbb{C}^{d}$ whenever $g \in \mathbb{C}^{d} \backslash\{0\}$.

If $A$ is a set we denote by $|A|$ its cardinal. For $f \in \mathbb{C}^{A}$ we represent by $\|f\|_{0}$ the cardinal of the set $\operatorname{supp} f=\{a \in A$ : $f(a) \neq 0\}$. Clearly, $\|\cdot\|_{0}$ is not a norm.

The first result we present was given in [15] as a quantitative version of a result of Krahmer et al. [14] which states that
$\left\|V_{g} f\right\|_{0} \geq d$ for every $f, g \in \mathbb{C}^{d} \backslash\{0\}$. Its proof was an adaptation of a method that was originally developed in [11, 12] in the continuous setting. We give a different proof improving the estimate.

Theorem 1 (Ghobber and Jaming [15]). Let $S \subset\{0,1, \ldots, d-$ $1\}^{2}$ be a subset with cardinal $|S|<d$. Then

$$
\|f\|\|g\| \leq \frac{1}{\sqrt{(1-(|S| / d))}}\left(\sum_{(j, k) \notin S}\left|\left(V_{g} f\right)(j, k)\right|^{2}\right)^{1 / 2} .
$$

Proof. From the definition we get

$$
\left|\left(V_{g} f\right)(j, k)\right| \leq \frac{1}{\sqrt{d}}\|f\|\|g\| .
$$

Hence

$$
\begin{aligned}
\|f\|^{2}\|g\|^{2}= & \sum_{(j, k) \notin S}\left|\left(V_{g} f\right)(j, k)\right|^{2} \\
& +\sum_{(j, k) \in S}\left|\left(V_{g} f\right)(j, k)\right|^{2} \\
\leq & \sum_{(j, k) \notin S}\left|\left(V_{g} f\right)(j, k)\right|^{2}+\frac{1}{d}\|f\|^{2}\|g\|^{2}|S|,
\end{aligned}
$$

from where the conclusion follows.

Our next aim is to investigate under which conditions the support of $V_{g} f$ has the smallest possible cardinal. The proof of the result that follows is based on an analysis of the discrete localization operators. These operators where introduced by Daubechies [20] in the continuous case in order to localize a signal both in time and frequency.

It is well known that $f$ can be recovered from $V_{g} f$ as

$$
f(\ell)=\|g\|^{-2} \frac{1}{\sqrt{d}} \sum_{(j, k) \in \mathbb{Z}_{d}^{2}}\left(V_{g} f\right)(j, k) g(\ell-j) e^{2 \pi i k \ell / d} .
$$

Definition 2. Let $g \in \mathbb{C}^{d}$ and $\Lambda \subset \mathbb{Z}_{d}^{2}$ be given such that $\|g\|=$ 1. Then, the localization operator $L_{\Lambda, g}: \mathbb{C}^{d} \rightarrow \mathbb{C}^{d}$ is defined by

$$
\left(L_{\Lambda, g} f\right)(\ell)=\frac{1}{\sqrt{d}} \sum_{(j, k) \in \Lambda}\left(V_{g} f\right)(j, k) g(\ell-j) e^{2 \pi i k \ell / d} .
$$

$L_{\Lambda, g} f$ is a filtered version of $f$, as only those values of $V_{g} f$ corresponding to entries in $\Lambda$ are considered. Clearly $L_{\Lambda, g} f=$ $f$ whenever $V_{g} f$ is supported in $\Lambda$. Let us denote by $\ell_{d}^{1}$ the vector space $\mathbb{C}^{d}$ endowed with the $\ell_{1}$-norm and by $\left\{e_{n}\right\}_{n=1}^{d}$ the canonical basis.

The main difficulty in the proof of the following theorem is that, a priori, $f$ and $g$ are arbitrary finite sequences of complex numbers. That is, we cannot assume that, after normalizing, $f(\ell)=e^{2 \pi i(u(\ell) / d)}$ and $g(\ell)=e^{2 \pi i(v(\ell) / d)}$ for some $\mathbb{Z}_{d}$-valued $u$ and $v$. 
Theorem 3. Let one assume that $\|f\|_{0}+\|g\|_{0}>d$. Then $\left\|V_{g} f\right\|_{0}=d$ if, and only if, $f(0) \neq 0$ and there are $a, \lambda, c$ such that $a^{d}=(-1)^{d-1}, \lambda^{d}=c^{d}=1$, and

$$
f(\ell)=a^{\ell} c^{\ell(\ell-1) / 2} f(0), \quad g(\ell)=(\lambda a)^{\ell} c^{\ell(\ell-1) / 2} g(0) .
$$

Proof. For every $j \in \mathbb{Z}_{d}$ the function $f \cdot \overline{T_{j} g}$ is different from zero; hence there is $k \in \mathbb{Z}_{d}$ such that $\left(V_{g} f\right)(j, k) \neq 0$. Consequently, the hypothesis $\left\|V_{g} f\right\|_{0}=d$ means that the support of $V_{g} f$ is a set

$$
\Lambda=\left\{\left(j, k_{j}\right): j \in \mathbb{Z}_{d}\right\}
$$

Without loss of generality we can assume that $\|g\|_{2}=1$. We now consider the discrete localization operator $L_{\Lambda, g}: \ell_{d}^{1} \rightarrow$ $\ell_{d}^{1}$

$$
\left(L_{\Lambda, g} h\right)(\ell)=\frac{1}{\sqrt{d}} \sum_{(j, k) \in \Lambda}\left(V_{g} h\right)(j, k) g(\ell-j) e^{2 \pi i k \ell / d} .
$$

It is well known that

$$
\begin{aligned}
& \left\|L_{\Lambda, g}: \ell_{d}^{1} \longrightarrow \ell_{d}^{1}\right\| \\
& \quad=\max _{n} \sum_{\ell=0}^{d-1}\left|\left(L_{\Lambda, g} e_{n}\right)(\ell)\right| \\
& \quad=\frac{1}{d} \max _{n} \sum_{\ell=0}^{d-1}\left|\sum_{j=0}^{d-1} \overline{g(n-j)} g(\ell-j) e^{-2 \pi i\left(k_{j}(n-\ell) / d\right)}\right| \\
& \quad \leq 1 .
\end{aligned}
$$

Since $L_{\Lambda, g} f=f$ then $\left\|L_{\Lambda, g}: \ell_{d}^{1} \rightarrow \ell_{d}^{1}\right\| \geq 1$ and we finally obtain

$$
\max _{n} \frac{1}{d} \sum_{\ell=0}^{d-1}\left|\left\langle g_{\ell}, g_{n}\right\rangle\right|=1
$$

where

$$
g_{\ell}(j)=g(\ell-j) e^{2 \pi i k_{j} \ell / d} .
$$

This implies that there is $\ell_{0} \in \mathbb{Z}_{d}$ such that

$$
\left|\left\langle g_{\ell}, g_{\ell_{0}}\right\rangle\right|=1 \quad \forall \ell \in \mathbb{Z}_{d}
$$

According to Cauchy-Schwarz inequality, for every $\ell \in \mathbb{Z}_{d}$ there is $\mu(\ell) \in \mathbb{C}$ such that $|\mu(\ell)|=1$ and $g_{\ell}=\mu(\ell) \cdot g_{\ell_{0}}$. That is,

$$
\frac{g(\ell-j)}{g\left(\ell_{0}-j\right)}=\mu(\ell) \cdot e^{-2 \pi i k_{j}\left(\ell-\ell_{0}\right) / d}, \quad \ell \in \mathbb{Z}_{d},
$$

which implies

$$
\frac{g(\ell-j)}{g(n-j)}=\frac{\mu(\ell)}{\mu(n)} \cdot e^{-2 \pi i k_{j}(\ell-n) / d} \quad \forall \ell, n, j .
$$

We now check that $\bar{\mu} \cdot f$ is constant. In fact, for every $n \in \mathbb{Z}_{d}$,

$$
\begin{aligned}
\left(V_{g} f\right)(j, k)= & \frac{1}{\sqrt{d}} \sum_{\ell=0}^{d-1} f(\ell) \overline{g(\ell-j)} e^{-2 \pi i k \ell / d} \\
= & \frac{\overline{g(n-j)}}{\overline{\mu(n)}} e^{-2 \pi i k_{j} n / d} \frac{1}{\sqrt{d}} \\
& \times \sum_{\ell=0}^{d-1} f(\ell) \overline{\mu(\ell)} e^{2 \pi i \ell\left(k_{j}-k\right) / d} \\
= & \frac{\overline{g(n-j)}}{\overline{\mu(n)}} e^{-2 \pi i k_{j} n / d} \widehat{\bar{\mu} \cdot f}\left(k-k_{j}\right) .
\end{aligned}
$$

Since the support of $V_{g} f$ coincides with $\Lambda$ we conclude that the Fourier transform of $\bar{\mu} \cdot f$ vanishes at any coordinate $k \epsilon$ $\mathbb{Z}_{d} \backslash\{0\}$. Hence $\bar{\mu} \cdot f$ is constant and we conclude

$$
\frac{g(\ell-j)}{g(n-j)}=\frac{\overline{f(n)}}{\overline{f(\ell)}} \cdot e^{-2 \pi i k_{j}(\ell-n) / d} \quad \forall \ell, n, j .
$$

After replacing $\ell, n$, and $j$ by $\ell+1, n+1$, and $j+1$ in the previous identity we obtain

$$
e^{-2 \pi i k_{j+1}(\ell-n) / d} \frac{\overline{f(n+1)}}{\overline{f(\ell+1)}}=e^{-2 \pi i k_{j}(\ell-n) / d} \frac{\overline{f(n)}}{\overline{f(\ell)}} .
$$

We take $\ell=n+1$ and conclude that

$$
e^{2 \pi i\left(k_{j+1}-k_{j}\right) / d}
$$

does not depend on $j$. That is, $k_{j+1}-k_{j}$ is constant (modulo d) and there is $p \in \mathbb{Z}$ such that

$$
k_{j+1}=k_{0}+p j \quad(\text { modulo } d) .
$$

We put $\lambda:=e^{-2 \pi i k_{0} / d}$ and take $n=j=0$ in (22) to obtain

$$
g(\ell) \cdot \overline{f(\ell)}=\lambda^{\ell} \overline{f(0)} g(0) .
$$

In order to simplify we normalize the function $f$ so that

$$
\overline{f(0)} g(0)=1 \text {. }
$$

We take $n=j$ in (22) to obtain

$$
g(\ell-j)=e^{-2 \pi i\left(k_{0}+p j\right)(\ell-j) / d} \frac{\overline{f(j)}}{\overline{f(\ell)}} g(0),
$$

that is,

$$
\begin{aligned}
& \frac{\lambda^{\ell-j}}{\overline{f(\ell-j)}}=\lambda^{\ell-j} e^{-2 \pi i p j(\ell-j) / d} \frac{\overline{f(j)}}{\overline{f(\ell)}} g(0), \\
& f(\ell)=f(\ell-j) f(j) e^{2 \pi i p j(\ell-j) / d} \overline{g(0)} .
\end{aligned}
$$

Then $j=1$ gives

$$
f(\ell)=f(\ell-1) f(1) e^{2 \pi i p(\ell-1) / d} \overline{g(0)} .
$$


Finally, we consider

$$
a:=f(1) \overline{g(0)}, \quad b:=\frac{\lambda}{\bar{a}}, \quad c:=e^{2 \pi i p / d} .
$$

Then

$$
f(\ell)=a c^{\ell-1} f(\ell-1) \quad \forall \ell \in \mathbb{Z}_{d},
$$

which implies

$$
f(\ell)=a^{\ell} c^{\ell(\ell-1) / 2} f(0) \quad \forall \ell \in \mathbb{Z}_{d} .
$$

Since $f(\ell)=f(\ell+d)$ we conclude

$$
a^{d} c^{d(d-1) / 2}=1
$$

That is, $a^{d}=(-1)^{d-1}$. Now, using that $|a|=|c|=1$,

$$
\begin{aligned}
g(\ell) & =\frac{\lambda^{\ell}}{\overline{f(\ell)}}=\left(\frac{\lambda}{\bar{a}}\right)^{\ell} c^{\ell(\ell-1) / 2} \frac{1}{\overline{f(0)}} \\
& =(\lambda a)^{\ell} c^{\ell(\ell-1) / 2} g(0) .
\end{aligned}
$$

The necessary condition is proved. In order to show the sufficiency, let us consider $a, \lambda, c$ with $\lambda^{d}=c^{d}=1, a^{d}=(-1)^{d-1}$ and

$$
f(\ell)=a^{\ell} c^{\ell(\ell-1) / 2}, \quad g(\ell)=(\lambda a)^{\ell} c^{\ell(\ell-1) / 2} .
$$

Then

$$
\begin{aligned}
\left(V_{g} f\right)(j, k) & =\frac{(\lambda a)^{j}}{\sqrt{d}} \sum_{\ell=0}^{d-1}(\bar{\lambda})^{\ell} c^{\left(2 \ell j-j^{2}-j\right) / 2} e^{-2 \pi i k \ell / d} \\
& =\frac{(\lambda a)^{j}}{\sqrt{d}} c^{\left(-j^{2}-j\right) / 2} \sum_{\ell=0}^{d-1} r_{j, k}^{\ell},
\end{aligned}
$$

where

$$
r_{j, k}=\bar{\lambda} c^{j} e^{2 \pi i k / d}
$$

Consequently,

$$
\left(V_{g} f\right)(j, k) \neq 0 \Longleftrightarrow r_{j, k}=1 .
$$

That is, using that $\bar{\lambda}=e^{2 \pi i m / d}$, we have that

$$
\left(V_{g} f\right)(j, k) \neq 0 \Longleftrightarrow k=m+j p \quad(\text { modulo } d)
$$

According to the previous result, the support of $V_{g} f$ is highly regular for instance when $g$ does not vanish and $\left\|V_{g} f\right\|_{0}=d$.

Next we prove that the periodicity of $f$ and $g$ is related to the fact that the short time Fourier transform $V_{g} f$ is supported in a certain subgroup.

Proposition 4. Let $d=p q$ and $f, g \in \mathbb{C}^{d} \backslash\{0\}$ be given. Then, the following conditions are equivalent.
(1) There is $\lambda \in \mathbb{C}$ such that $\lambda^{p}=1$ and

$$
f(\ell+q)=\lambda f(\ell), \quad g(\ell+q)=\lambda g(\ell), \quad \ell \in \mathbb{Z}_{d}
$$

(2) The support of $V_{g} f$ is contained in $\mathbb{Z}_{d} \times p \mathbb{Z}_{d}$.

Proof. (1) implies (2) is obvious and we only prove (2) implies (1). Without loss of generality, we can assume that $\|g\|_{2}=1$. We take $\Lambda=\mathbb{Z}_{d} \times p \mathbb{Z}_{d}$ and consider the localization operator $L_{\Lambda, g}: \ell_{d}^{1} \rightarrow \ell_{d}^{1}$ defined by

$$
\left(L_{\Lambda, g} h\right)(\ell)=\frac{1}{\sqrt{d}} \sum_{(j, k) \in \Lambda}\left(V_{g} h\right)(j, k) g(\ell-j) e^{2 \pi i k \ell / d} .
$$

Condition (2) implies $L_{\Lambda, g} f=f$; hence

$$
\left\|L_{\Lambda, g}: \ell_{d}^{1} \longrightarrow \ell_{d}^{1}\right\| \geq 1 .
$$

That is,

$$
\max _{n} \sum_{\ell=0}^{d-1}\left|\left(L_{\Lambda, g} e_{n}\right)(\ell)\right| \geq 1
$$

On the other hand, $\left(V_{g} e_{n}\right)(j, k)=\frac{1}{\sqrt{d}} \overline{g(n-j)} e^{-2 \pi i(n k / d)}$ and

$$
\left(L_{\Lambda, g} e_{n}\right)(\ell)=\frac{1}{d}\left(\sum_{j=0}^{d-1} \overline{g(n-j)} g(\ell-j)\right)
$$

$$
\times\left(\sum_{k=0}^{q-1} e^{-2 \pi i((n-\ell) k / q)}\right)
$$

Consequently,

$$
\left(L_{\Lambda, g} e_{n}\right)(\ell)=0 \text { whenever } n \neq \ell \text { modulo } q \text {, }
$$

$$
\left(L_{\Lambda, g} e_{n}\right)(\ell)=\frac{1}{p} \sum_{j=0}^{d-1} \overline{g(n-j)} g(\ell-j)
$$

$$
\text { whenever } n=\ell \text { modulo } q \text {. }
$$

From (44), Cauchy-Schwarz inequality, and condition $\|g\|_{2}=$ 1 we finally conclude that, for some $n \in \mathbb{Z}_{d}$,

$$
\sum_{m=0}^{p-1} \frac{1}{p}\left|\sum_{j=0}^{d-1} \overline{g(n-j)} g(n+m q-j)\right|=1
$$

Hence

$$
\begin{array}{r}
\left|\sum_{j=0}^{d-1} \overline{g(n-j)} g(n+m q-j)\right|=1 \\
\quad \text { for every } m=0,1, \ldots, p-1 .
\end{array}
$$

In particular, by Cauchy-Schwarz inequality, there is $\lambda \in \mathbb{C}$ with $|\lambda|=1$ such that

$$
g(n+q-j)=\lambda g(n-j), \quad j=0,1, \ldots, d-1 .
$$


That is, $g(\ell+q)=\lambda g(\ell)$ for every $\ell=0,1, \ldots, d-1$. From $g(\ell)=g(\ell+d)=\lambda^{p} g(\ell)$ we obtain $\lambda^{p}=1$. Since $\left|V_{g} f(j, k)\right|=$ $\left|V_{f} g(-j,-k)\right|$ we can proceed as before and obtain $f(\ell+q)=$ $\mu f(\ell)$ for some $\mu \in \mathbb{C}$ with $\mu^{p}=1$. Finally, from the fact that

$$
\begin{aligned}
\left(V_{g} f\right)(j, k)= & \frac{1}{\sqrt{d}}\left(\sum_{\ell=0}^{q-1} f(\ell) \overline{g(\ell-j)} e^{-2 \pi i(\ell k / q)}\right) \\
& \times\left(\sum_{m=0}^{p-1}(\bar{\lambda} \mu)^{m} e^{-2 \pi i(m k / p)}\right)
\end{aligned}
$$

is different from zero for some $k \in p \mathbb{Z}_{d}$ we conclude $\bar{\lambda} \mu=1$. That is, $\lambda=\mu$ and the proposition is proved.

In connection with the norm attaining points of the Fourier transform the following definition was given in [16].

Definition 5. We say that $f=\left(f_{i}\right)_{i=0}^{d-1} \in \mathbb{C}^{d}$ gives rise to a time-frequency basis if the translations of $(1 / \sqrt{d}) f$ form an orthonormal basis and this basis is equal to $\left\{c_{k}(1 / \sqrt{d}) M_{k} f\right.$ : $\left.k \in \mathbb{Z}_{d}\right\}$ for some constants $c_{k} \in \mathbb{T}$.

If $f$ generates a time-frequency basis it must be biunimodular; that is, $\left|f_{i}\right|=\left|\widehat{f}_{i}\right|=1$, but the opposite does not hold. Biunimodular vectors and vectors generating timefrequency basis are easily characterized in terms of the support of $V_{f} f$.

Proposition 6. Let $f \in \mathbb{C}^{d} \backslash\{0\}$ be given. Then

(1) $f$ is biunimodular if and only if $V_{f} f(0, k)=V_{f} f(k$, $0)=\delta_{k, 0}$,

(2) a multiple of $f$ generates a time-frequency basis if and only if

$$
\operatorname{supp} V_{f} f=\{(j, \pi(j)): j=0, \ldots, d-1\},
$$

where $\pi:\{0, \ldots, d-1\} \rightarrow\{0, \ldots, d-1\}$ is a permutation.

Proof. (1) Follows from $V_{f} f(0, k)=\widehat{|f|^{2}}(k)$ and $V_{\widehat{f}} \widehat{f}(j, k)=$ $V_{f} f(k, j)$.

(2) Let us first assume that $f \in \mathbb{C}^{d}$ generates a time-frequency basis. Accordingly, $\left|f_{i}\right|=1$ and there is a permutation $\pi:\{0, \ldots, d-1\} \rightarrow\{0, \ldots, d-1\}$ such that $\pi(0)=0$ and

$$
f(\ell-j)=\lambda_{j} e^{-2 \pi i \pi(j) \ell / d} f(\ell), \quad\left|\lambda_{j}\right|=1 .
$$

Then

$$
\left(V_{f} f\right)(j, k)=\frac{\overline{\lambda_{j}}}{\sqrt{d}} \sum_{\ell=0}^{d-1} e^{-2 \pi i(k-\pi(j)) / d}
$$

which is nonzero only for $k=\pi(j)(\bmod d)$.

To show the converse implication we first note that $\left(V_{f} f\right)(0,0)=(1 / \sqrt{d})\|f\|^{2}>0$ implies $\pi(0)=0$. As $V_{f} f(j$, $k)=\widehat{f \overline{T_{j} f}}(k)$ one has

$$
\widehat{f \overline{T_{j} f}}=\lambda_{j} e_{\pi(j)}
$$

for some $\lambda_{j} \in \mathbb{C}$, from where

$$
f(\ell) \overline{f(\ell-j)}=\mu_{j} e^{2 \pi i \ell \pi(j) / d}
$$

for some $\mu_{j} \in \mathbb{C}$. Since $\pi(0)=0$ then $|f(\ell)|^{2}=\mu_{0}$. Therefore $|f(\ell)|$ is constant. We now deduce

$$
\left|\mu_{j}\right|=|f(\ell) f(\ell-j)|=\mu_{0}
$$

for every $j$. In particular, we can write

$$
\mu_{1}=\mu_{0} e^{-2 \pi i r_{1}}
$$

and we obtain

$$
f(\ell) \overline{f(\ell-1)}=\mu_{0} e^{-2 \pi i r_{1}} e^{2 \pi i \ell \pi(1) / d}, \quad \ell \in \mathbb{Z}_{d} .
$$

Proceeding by recurrence we finally obtain

$$
f(\ell)=f(0) e^{-2 \pi i\left(r_{1} \ell+k_{1}(\ell+1) \ell\right) / 2 d},
$$

and then it is easy to see that for some constants $c_{j} \in \mathbb{T}, T_{j} f=$ $c_{j} M_{j k_{1}} f$. Finally, as $V_{f} f(j, 0)=0$ for $j \neq 0$ we get that $\left\{T_{j} f\right.$ : $j=1, \ldots, d-1\}$ is an orthogonal basis, and this basis coincides up to constants with the set of all modulations.

As an application we recover [16, Theorem 4.5].

Corollary 7. Let $f \in \mathbb{C}^{d} \backslash\{0\}$ be given. Then a multiple of $f$ generates a time-frequency basis if and only if there exist $a$ and $c$ such that $a^{d}=(-1)^{d-1}, c$ is a primitive d-root of unity, and

$$
f(\ell)=a^{\ell} c^{\ell(\ell-1) / 2} f(0), \quad \ell \in \mathbb{Z}_{d}
$$

Proof. Let us assume that $f$ generates a time-frequency basis and $f(0)=1$. According to Theorem 3, there exist $a$ and $c$ such that $a^{d}=(-1)^{d-1}, c^{d}=1$, and

$$
f(\ell)=a^{\ell} c^{\ell(\ell-1) / 2} f(0), \quad \ell \in \mathbb{Z}_{d}
$$

We put $c=e^{-2 \pi i p / d}$. From the proof of the same result we have $\left(V_{f} f\right)(j, k) \neq 0$ if and only if $k=p j$ (modulo $d$ ). Hence, Proposition 6 gives that the map $\mathbb{Z}_{d} \rightarrow \mathbb{Z}_{d}, j \mapsto p j$, is injective, or equivalently, $p$ and $d$ have no common prime divisors. That is, $c$ is a primitive $d$-root of unity.

Corollary 8. Let $d$ be a prime number. Then $\left\|V_{f} f\right\|_{0}=d$ if and only if $f$ satisfies one of the following conditions:

(1) $\|f\|_{0}=1$.

(2) $\|\widehat{f}\|_{0}=1$.

(3) (A multiple of) $f$ generates a time-frequency basis.

Proof. We assume $d>2$. If $2\|f\|_{0}>d$ we can apply Theorem 2.5 to conclude that $f(\ell)=f(0) a^{\ell} c^{\ell(\ell-1) / 2}$ with $a^{d}=c^{d}=1$. If $c \neq 1$ it is a primitive $d$-root of the unity and therefore $f$ generates a time-frequency basis. In case $c=1,\|\widehat{f}\|_{0}=1$.

If $2\|f\|_{0} \leq d$ then, by [6], we have $2\|\widehat{f}\|_{0}>d$; hence as before either $\widehat{f}$ generates a time-frequency basis or the support of $\widehat{f}$ is a singleton. 


\section{Conflict of Interests}

The authors declare that there is no conflict of interests regarding the publication of this paper.

\section{Acknowledgments}

The research of C. Fernández and A. Galbis was partially supported by MEC and FEDER Projects nos. MTM201015200 and GVA Prometeo no. II/2013/013. The research of J. Martínez was supported by MEC Project no. MTM200804594.

\section{References}

[1] G. B. Folland and A. Sitaram, "The uncertainty principle: a mathematical survey," The Journal of Fourier Analysis and Applications, vol. 3, no. 3, pp. 207-238, 1997.

[2] D. L. Donoho and P. B. Stark, "Uncertainty principles and signal recovery," SIAM Journal on Applied Mathematics, vol. 49, no. 3, pp. 906-931, 1989.

[3] R. Meshulam, "An uncertainty inequality for groups of order pq," European Journal of Combinatorics, vol. 13, no. 5, pp. 401407, 1992.

[4] E. Matusiak, M. Özaydın, and T. Przebinda, "The DonohoStark uncertainty principle for a finite abelian group," Acta Mathematica Universitatis Comenianae. New Series, vol. 73, no. 2, pp. 155-160, 2004.

[5] K. T. Smith, "The uncertainty principle on groups," SIAM Journal on Applied Mathematics, vol. 50, no. 3, pp. 876-882, 1990.

[6] T. Tao, "An uncertainty principle for cyclic groups of prime order," Mathematical Research Letters, vol. 12, no. 1, pp. 121-127, 2005.

[7] R. Meshulam, "An uncertainty inequality for finite abelian groups," European Journal of Combinatorics, vol. 27, no. 1, pp. 63-67, 2006.

[8] A. Bonami, B. Demange, and P. Jaming, "Hermite functions and uncertainty principles for the Fourier and the windowed Fourier transforms," Revista Matemática Iberoamericana, vol. 19, no. 1, pp. 23-55, 2003.

[9] B. Demange, "Uncertainty principles for the ambiguity function," Journal of the London Mathematical Society. Second Series, vol. 72, no. 3, pp. 717-730, 2005.

[10] C. Fernández and A. Galbis, "Annihilating sets for the short time Fourier transform," Advances in Mathematics, vol. 224, no. 5, pp. 1904-1926, 2010.

[11] P. Jaming, "Principe d'incertitude qualitatif et reconstruction de phase pour la transformée de Wigner," Comptes Rendus de l'Académie des Sciences. Série I. Mathématique, vol. 327, no. 3, pp. 249-254, 1998.

[12] A. J. E. M. Janssen, "Proof of a conjecture on the supports of Wigner distributions," The Journal of Fourier Analysis and Applications, vol. 4, no. 6, pp. 723-726, 1998.

[13] E. Wilczok, "New uncertainty principles for the continuous Gabor transform and the continuous wavelet transform," Documenta Mathematica, vol. 5, pp. 201-226, 2000.

[14] F. Krahmer, G. E. Pfander, and P. Rashkov, "Uncertainty in time-frequency representations on finite abelian groups and applications," Applied and Computational Harmonic Analysis, vol. 25, no. 2, pp. 209-225, 2008.
[15] S. Ghobber and P. Jaming, "On uncertainty principles in the finite dimensional setting," Linear Algebra and its Applications, vol. 435, no. 4, pp. 751-768, 2011.

[16] J. Gilbert and Z. Rzeszotnik, "The norm of the Fourier transform on finite abelian groups," Annales de l'Institut Fourier, vol. 60, no. 4, pp. 1317-1346, 2010.

[17] H. G. Feichtinger, W. Kozek, and F. Luef, "Gabor analysis over finite abelian groups," Applied and Computational Harmonic Analysis, vol. 26, no. 2, pp. 230-248, 2009.

[18] K. Gröchenig, Foundations of Time-Frequency Analysis, Applied and Numerical Harmonic Analysis, Birkhäuser, Boston, Mass, USA, 2001.

[19] K. Gröchenig, "Uncertainty principles for time-frequency representations," in Advances in Gabor analysis, pp. 11-30, Birkhäuser, Boston, Mass, USA, 2003.

[20] I. Daubechies, “Time-frequency localization operators: a geometric phase space approach," IEEE Transactions on Information Theory, vol. 34, no. 4, pp. 605-612, 1988. 


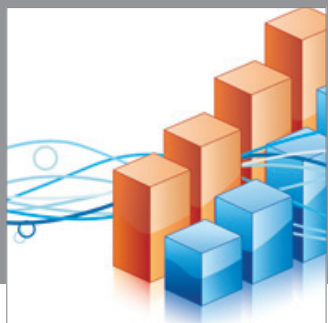

Advances in

Operations Research

mansans

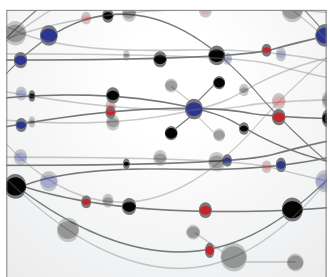

The Scientific World Journal
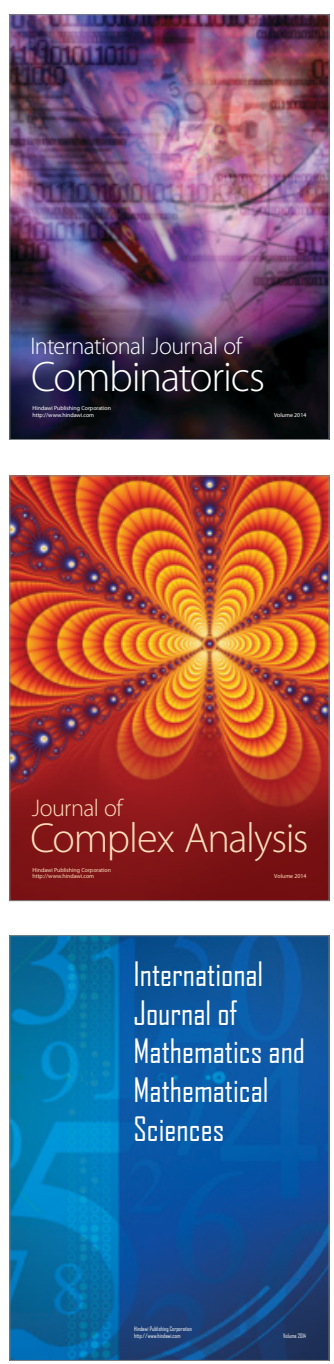
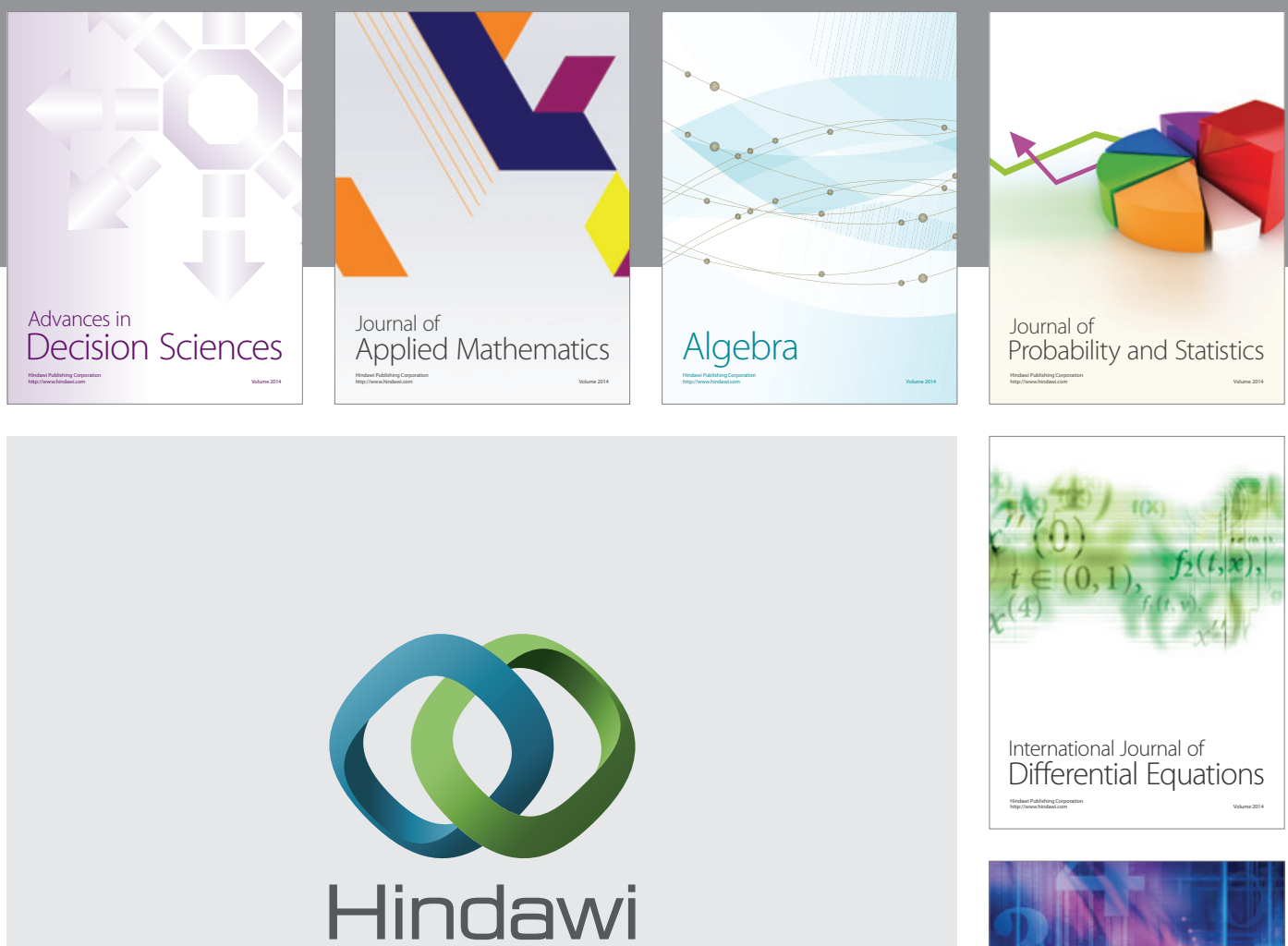

Submit your manuscripts at http://www.hindawi.com
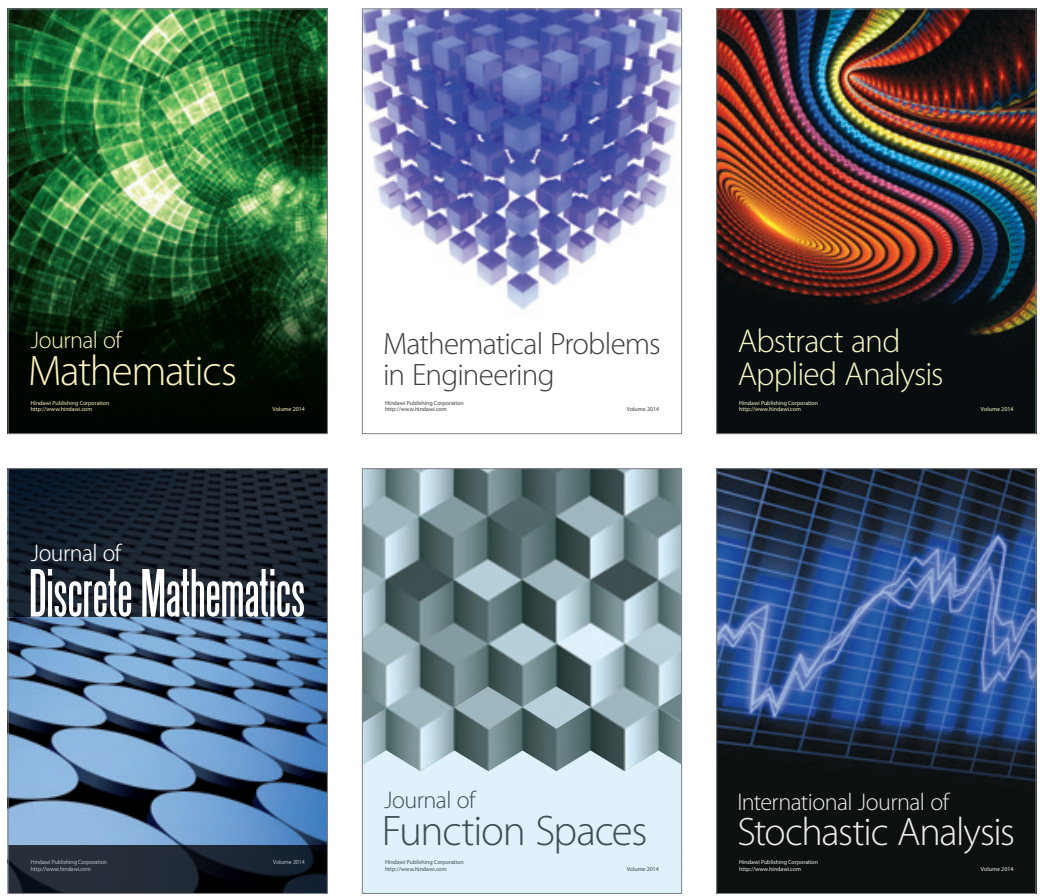

Journal of

Function Spaces

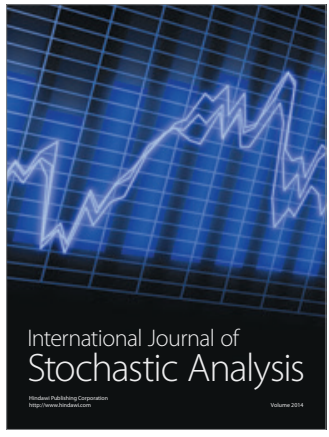

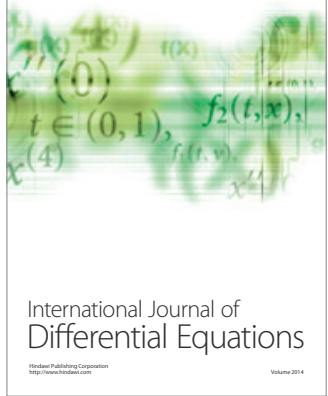
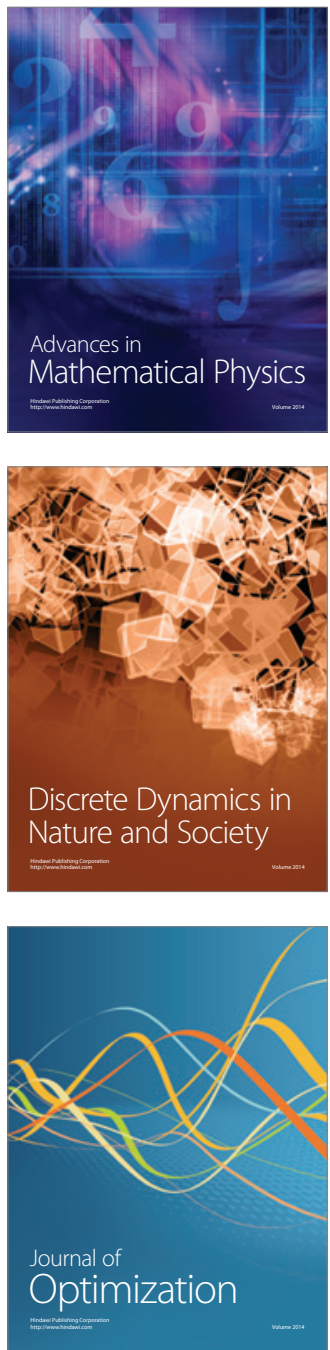